\title{
Impact of COVID-19 Pandemic on Disability Care and Persons with Disability in Pakistan
}

\author{
Farooq Azam Rathore ${ }^{1}$ and Ahmad Zaheer Qureshi ${ }^{2}$ \\ ${ }^{1}$ Department of Rehabilitation Medicine, Armed Forces Institute of Rehabilitation Medicine, Rawalpindi, Pakistan \\ ${ }^{2}$ Department of Physical Medicine and Rehabilitation, King Fahad Medical City, Riyadh, Saudi Arabia
}

December 2021 marks the second anniversary of the detection of the COVID-19 virus from Wuhan, China, which resulted in a global healthcare crisis, leading to a pandemic. It impacted mobility, halted economic activities, led to prolonged closure of educational institutes, religious places, repeated lockdowns and a huge burden of morbidity and mortality. However, people are trying to gradually adapt to the post-COVID-19 world and moving on with their daily chores. The impact of COVID on healthcare systems, economies and individual patients has been well documented in the literature, and continues to expand. This includes documentation of the impact of COVID-19 on persons with disability (PWD). But, almost all the published literature is from the West and the high-income countries with few publications from the low and low-middle income country(ies) (LIC/LMIC). The adverse effects of COVID-19 pandemic on the PWD in Pakistan; a LMIC, also need to be highlighted, and suggest recommendations to address this gap in healthcare delivery.

According to the United Nations, "Persons with disabilities in the world are among the hardest hit by COVID-19."1 Many reasons have been identified for the increased risk of PWD contracting COVID-19. It is difficult for PWD to follow the guidelines issued for checking the spread of the virus like washing hands or social distancing. Many PWD in the remote areas do not have ready access to water and sanitation; and social distances might not be even possible, especially when they are dependent upon others for their mobility and transfer needs. PWD are at a higher risk of developing obesity, lung and cardiac problems, due to the nature of their disability and reduced mobility. When these PWD with comorbidities contract COVID-19, their risk of severe infection and subsequent mortality increases. PWD in LMIC, like Pakistan, faced discrimination in accessing healthcare services even before the COVID-19 pandemic. For example, during the earthquake of 2005 and subsequent relief activities, able bodied persons were able to access and carry the medications and relief items, while the PWD were unable to move and thus were deprived.

Correspondence to: Dr. Farooq Azam Rathore, Department of Rehabilitation Medicine, Armed Forces Institute of Rehabilitation Medicine, Rawalpindi, Pakistan

E-mail: farooqrathore@gmail.com

Received: October 03, 2021; Revised: October 20, 2021;

Accepted: November 01, 2021

DOI: https://doi.org/10.29271/jcpsp.2021.12.1391
There is evidence that PWD have also faced increased inter-personal violence in the wake of COVID-19-related lockdowns. ${ }^{1}$ The situation is further complicated by the fact that the PWD usually rely on the perpetrator for care and assistance; and there are multiple barriers to reporting abuse and seeking help, including lack of a clear mechanism for reporting. In addition, there might be a fear of retaliation and other negative consequences, if abuse is reported. ${ }^{2}$

The PWD in Pakistan were ignored even before the COVID-19 pandemic. They were usually not part of the mainstream society, thus faced many social, cultural and mobility barriers. According to the $6^{\text {th }}$ population and housing census of 2017, the population of Pakistan is around 207 million. ${ }^{3}$ Considering the World Health Organization (WHO) estimate of 15 percent prevalence of global disability, ${ }^{4}$ around 31 million people in Pakistan are expected to be living with some form of disability. But there are no reliable statistics of disable persons in Pakistan. ${ }^{3}$

While COVID-19 adversely affected everyone, in one way or the other, the impact was particularly severe for PWD. Due to the closure of routine medical services, both in the public and private sectors, many PWD were denied the rehabilitation services essential for their recovery and prevention of complications (for example rehabilitation medicine consultation, physical therapy, occupational therapy, and provision of orthotics). The access to essential medicines for a PWD, e.g., anti-spasticity drugs and medications for neuropathic pain, were also disrupted. This has resulted in worsening of disability, reduced mobility, development of many preventable complications, and deterioration in quality of life.

While the healthcare professionals diverted all their attention to the management of COVID-19, the PWD were ignored with no clear plans or guidelines to help them out during these difficult times. The number of cases developing new disabilities like stroke, multiple sclerosis, long bone fractures, brain trauma, neurodegerative disorders and other chronic pain syndromes, keptonincreasing.

The Government of Pakistan has issued more than 90 guidelines regarding different aspects of COVID-19, like quarantine, testing, use of protective equipment, vaccinations, treatment options, preventive measures, and burial of the dead. ${ }^{5}$ However, till October 2021, the Government issued no guidelines on how to address disabilities arising from COVID-19 and to support those with pre-existing disabilities during this pandemic. 
Collateral impact of COVID-19 on disability care is another aspect, which is yet to be explored. One of the collateral effects is the repercussions of COVID-19 pandemic on the care of individuals with disability having no COVID-19 infection. Similarly, long term sequel of COVID-19 is not well established uptill now.

The vulnerable population includes the people who encounter new onset disabilities, like traumatic injuries, strokes, or disabling musculoskeletal problems, and have limited resources to seek rehabilitation care. Likewise, patients who have chronic illnesses and are confined to bed due to total dependency, are another group of patients at risk to develop debilitating complications during the COVID pandemic. The number of individuals seeking rehabilitation care decreased during pandemic, but that should not mean that the new onset disabilities are not occurring. It is reported that upto 2.2 million people experiencing disability suffer collateral damage each day due to COVID-19 lockdown in Europe. ${ }^{6}$ There are many possibilities, which can explain these collateral effects; as there is reluctance of going to the hospital for seeking medical or rehabilitation care due to fear of acquiring COVID-19 infection. ${ }^{7}$ This poses a challenge for individuals requiring disability care since they generally do not have acute issues, and may end up postponing care leading to complications of prolonged immobility, like spasticity, contractures, malnourishment, skin breakdown, mood impairments, infections, falls, sleep disturbances, cardiovascular complications; and subsequently, delayed or hampered functional recovery. This is not limited to physical complications, but a considerable impact of psychological disorders adds on to the mental challenges faced by the patients due to new onset disabilities. It is important to note that it is difficult to identify and report cases who undergo social isolation, and are vulnerable to neglect and abuse, especially the elderly and the children, who are totally dependent on others. This reflects on the financial constraints and social pressure on the families or caregivers, who may be under a distressing situation because of the socio-economic impact of COVID. During the pandemic, the hospital resources are channelised to acute care, indirectly impacting the provision of bare minimum resources needed by individuals with disabilities to be in continuous care at home, such as equipment including wheel chair, hospital beds, commode chairs, oxygen supplies and hygiene products, which are needed by individuals with disabilities on day-to-day basis. On the other hand, patients with disabilities such as stroke, who stayed longer in the hospitals during the COVID-19 pandemic due to non-COVID-related issues such as discharge disposition challenges, continue to remain at risk of COVID exposure during hospitalisation. ${ }^{8}$

Institutional factors play a critical role in handling individuals with disability due to non-COVID causes. The main determinant is, if the facility is involved in COVID care or not as many rehabilitation centres underwent dramatic transformations to accommodate patients with COVID-19. ${ }^{9}$ Some of the other institutional challenges include limited active rehabilitation services, challenges in sustaining medical supplies, cohorting patients and healthcare providers, gyms and space utilisation, equipment sterilisation and exposure tracing.
Access to rehabilitation facilities has been one of the major challenges in acquiring opportunity for early rehabilitation, which remains important in neurological disabilities such as stroke, spinal cord injuries, traumatic brain injuries, where onset of rehabilitation plays a critical role in functional outcomes. Travel restrictions and a busy emergency response service, limited the opportunities of care needed by individuals with disabilities, when needed. It is important consideration that individuals with disabilities have already limited access to specialised care services, with their disabilities further limiting their access to care independently. During COVID pandemic, the need to maintain essential rehabilitation services goes unrecognised in many healthcare systems. ${ }^{10}$

There is a need to increase the awareness about disability care during pandemic among healthcare providers and administrative stakeholders, so the special needs of this vulnerable group of patients do not go unnoticed. The COVID pandemic is an eyeopener for health systems to plan beyond pandemic and work towards readiness for disaster situations, including, natural emergencies or human-induced disasters. Rehabilitation centres in different regions of Pakistan should be identified from now, which can provide uninterrupted rehabilitation care throughout a national emergency. Development and standardisation of telerehabilitation services at national level must be instituted. Institutional guidelines should not only be focused on COVID care, rather should include recommendations and action plans to sustain parallel care to individuals with disabilities. National and international disability organisations, operating in Pakistan, need to advocate the cause at the fora of World Health Organisation and United Nations, to specially allocate resources for low and middle income countries for disability care during disaster management.

\section{REFERENCES}

1. United Nations. International Day of Persons with Disabilities, 3 December Available from http://www. un.org/en/observances/day-of-persons-with-disabilities [Accessed on 29th Sept 2021].

2. Lund EM. Interpersonal violence against people with disabilities: Additional concerns and considerations in the COVID-19 pandemic. Rehabil Psychol 2020; 65(3): 199-205. doi: 10.1037/rep0000347.

3. Pakistan Bureau of Statistics. Population Census. Available from http://www.pbs.gov.pk/content/ population-census [Accessed on 7th September 2021].

4. World Bank. World report on disability: Main report (English). Washington, DC: World Bank. 2011 Available from http: documents.worldbank.org/curated/en/66513 1468331271288/Main-report [Accessed on 7th September 2021].

5. Government of Pakistan. Guidelines. Available at http://www.covid.gov.pk/guideline [Accessed on 29 Sept 2021].

6. Negrini S, Grabljevec K, Boldrini P, Kiekens C, Moslavac 
S, Zampolini M, et al. Up to 2. 2 million people experiencing disability suffer collateral damage each day of COVID-19 lockdown in Europe. Eur J Phys Rehabil Med 2020; 56(3):361-5. doi: 10.23736/S1973-9087. 20.06361-3.

7. Czeisler MÉ, Marynak K, Clarke KEN, Salah Z, Shakya I, Thierry JM, et al. Delay or Avoidance of Medical Care Because of COVID-19-Related Concerns - United States, June 2020. MMWR Morb Mortal Wkly Rep 2020; 69(36):1250-7. doi: 10.15585/mmwr.mm6936a4.

8. Abbas M, Robalo Nunes T, Martischang R, Zingg W, Iten $A$, Pittet $D$, et al. Nosocomial transmission and outbreaks of coronavirus disease 2019: The need to protect both patients and healthcare workers. Antimicrob Resist Infect Control 2021; 10(1):7. doi: 10.1186/s13756020-00875-7.

9. Mansoor SN, Gill ZA, Rathore FA, Uttra KM. Establishing and managing a quarantine and isolation centre in COVID-19 pandemic. J Pak Med Assoc 2020; 70(Suppl 3) (5):S11-S14. doi: 10.5455/JPMA.06.

10. Bettger JP, Thoumi A, Marquevich V, De Groote W, Rizzo Battistella L, Imamura M, et al. COVID-19: Maintaining essential rehabilitation services across the care continuum. BMJ Glob Health 2020; 5(5):e002670. doi: 10.1136/bmjgh-2020-002670. 